\title{
IMPLEMENTAÇÃO E CONTROLE DE RETIFICADOR TRIFÁSICO DE ALTA QUALIDADE COM COMUTAÇÃO EM BAIXA FREQÜÊNCIA
}

\author{
Joanna A. G. Marafão \\ DSCE - FEEC - UNICAMP \\ C.P. 6101 \\ 13081-970 Campinas - SP \\ Brasil
}

\begin{abstract}
Resumo - A implementação de um retificador trifásico de $10 \mathrm{~kW}$ de alta qualidade, baseado na ponte retificadora convencional com a adição de uma célula de comutação é apresentada neste artigo. Comparado com outros retificadores comutados na freqüência da linha, o circuito proposto não viola os limites de distorção da corrente, definidos na recomendação técnica IEC 61000-3-4, em nenhum nível de potência. A topologia permite ainda o controle da tensão de saída, regulando-a contra variações na carga e na entrada. $O$ controle é feito com o auxilio de um microcontrolador PIC17c756. Foi construído um protótipo e os resultados confirmam a análise teórica.
\end{abstract}

Abstract - This article presents the implementation of a $10 \mathrm{~kW}$ high-quality three-phase diode rectifier, based on a conventional rectifier with an add-on cell with linefrequency commutated AC switches. As compared to other line-frequency commutated rectifiers, the proposed circuit allows compliance with the low-frequency harmonic limits defined in the technical report IEC 61000-3-4 for any power range. Additionally it is possible to control the output voltage, rejecting load and input voltage variations. The control was made with the microcontroler PIC17c756. A prototype was built and tested. The results confirm the theoretical analysis.

\section{INTRODUÇÃO}

Estudos vêm demonstrando que é cada vez maior o percentual de cargas elétricas que utilizam algum tipo de conversor eletrônico de potência. Estimativas indicam que aproximadamente $50 \%$ de toda a energia elétrica consumida passe por algum tipo de processamento eletrônico [1].

Em grande parte destas cargas, sejam industriais ou residenciais, tem-se a presença de um estágio retificador na conexão com a rede, com a alimentação da carga sendo feita a partir da tensão contínua obtida em sua saída (conversores de frequiência, UPSs, equipamentos de informática, áudio, vídeo, iluminação, etc.).

A estrutura mais simples para tais retificadores, desde que não seja necessário um ajuste no valor da tensão contínua, é a de um retificador com um filtro capacitivo. No entanto, sabese que tais circuitos, sejam eles monofásicos ou trifásicos, apresentam um baixo fator de potência (em torno de 0,6 ) e distorção harmônica na corrente que em muito excede os limites estabelecidos por normas ou recomendações internacionais, como IEC 61000-3-2 [2] e IEC 61000-3-4 [3].

Indutores e capacitores podem ser utilizados em conjunto

\author{
Giorgio Spiazzi \\ Universidade de Pádua \\ Via Gradenigo 6/a, \\ 35131 Padova - PD \\ Itália
}

com pontes retificadoras para melhorar a forma de onda da corrente de entrada. A simples adição de um indutor na entrada da ponte de diodos já causa uma melhora: diminui a distorção da forma de onda da corrente e o fator de potência se eleva.

Os atrativos do uso de filtros LC para melhorar o fator de potência são sua simplicidade, baixas perdas e confiabilidade, devido à ausência de elementos ativos. No entanto, também apresentam inúmeras desvantagens que limitam sua aplicação, como resposta dinâmica pobre, são pesados e volumosos, não possibilitam regulação de tensão, afetam as formas de onda na frequiência fundamental e seu correto dimensionamento não é simples.

As soluções ativas são mais vantajosas. Topologias com chaveamento em alta frequiência levam a uma drástica redução nos valores dos elementos passivos (indutores e capacitores) utilizados, mas podem produzir problemas de interferências eletromagnéticas.

As topologias com chaveamento em baixa freqüência apresentam uma redução nas perdas por comutação e produzem menos interferência eletromagnética. Embora os elementos magnéticos sejam maiores dos que os usados nos retificadores com comutação em alta freqüência, é possível que o volume total do retificador seja menor, pela minimização de filtros de EMI (do inglês Electromagnetic Interference) e pela minimização dos dissipadores de calor [4].

Uma das técnicas para minimização da distorção da corrente é a injeção de uma corrente na freqüência do terceiro harmônico na entrada do retificador [5-7], a qual resulta numa forma de onda com um valor de distorção harmônica total (THD, Total Harmonic Distortion) próxima a 5\%.

Em [5] tal corrente de entrada é gerada no estágio CC do retificador, através de dois conversores tipo boost. A injeção de terceiro harmônico na entrada é feita através de três ramos L-C. Esta mesma topologia pode ser usada como inversor [6]. Porém apresenta algumas desvantagens: em aplicações de potência elevada, os elementos magnéticos podem apresentar um volume grande e existe a possibilidade de ocorrer uma ressonância devido à interação entre a impedância da rede e a do ramo L-C.

Com a utilização de um transformador Zig-zag [7] para fazer a injeção de terceiro harmônico, afasta-se a possibilidade de ressonância. A principal vantagem desta topologia é que sua operação independe da impedância da linha, mas exige um transformador especial.

Em [8], tem-se um retificador de 12 pulsos com um autotransformador com dois enrolamentos por fase. Novamente tem-se um elemento magnético relativamente 
complexo sem possibilidade de controle da tensão de saída.

Em [9] tem-se um circuito que permite, sem alterações significativas na topologia básica do retificador, elevar o fator de potência. A idéia é forçar a existência de uma corrente na fase que estaria desconectada sem alterar o comportamento da saída. No entanto, aparecem picos de tensão sobre os componentes nos momentos de abertura dos interruptores, devido ao desequilíbrio instantâneo das correntes, os quais devem ser limitados por circuitos snubber. Outra desvantagem é a necessidade da alimentação ser feita com neutro acessível, pelo qual circula uma corrente de terceiro harmônico de amplitude significativa.

Visando eliminar alguns destes problemas, foi desenvolvido o conversor Curi [10]. A principal diferença é que neste caso existe um caminho para a corrente presente nos indutores quando o interruptor deixa de conduzir. A continuidade se dá pela condução dos diodos da ponte retificadora trifásica, passando pelos capacitores de filtro. $\mathrm{O}$ capacitor de saída deve ser substituído por dois capacitores, criando um ponto médio, ou seja, há necessidade de intervenção na topologia.

Topologicamente o conversor Curi é idêntico ao retificador apresentado em [11]. A diferença entre ambos é a comutação, que em [11] é feita em alta freqüência.

Este trabalho propõe o desenvolvimento de um retificador com uma estrutura similar àquela apresentada em [10], mas que assegura o atendimento das limitações impostas pela IEC 61000-3-4, além de não exigir a alteração na topologia de retificadores já existentes. Na presente proposta, os indutores de entrada realizam uma ressonância com os capacitores adicionados no barramento $\mathrm{CC}$, o que reduz o conteúdo harmônico da corrente de entrada, para valores abaixo do limite recomendado pela IEC 61000-3-4 para qualquer nível de potência. No conversor Curi, como os capacitores são de valor muito maior, os mesmos se comportam como fontes de tensão, não se verificando formas de onda suavizadas, típicas das ressonâncias, de maneira que os limites de [3] nunca são atendidos para algumas ordens harmônicas.

Outro aspecto refere-se à aplicação de uma carga que absorva corrente pulsada em alta freqüência. No conversor Curi esta corrente circularia pelos dois capacitores, enquanto na topologia proposta ocorre um desacoplamento dos estágios de entrada e saída, concentrando-se os componentes de alta frequiência no capacitor de saída, minimizando a propagação de ruído para a rede.

A topologia proposta está apresentada na seção II. Nas seções III e IV tem-se a descrição da construção do protótipo e a implementação dos circuitos responsáveis pela estrutura de acionamento e controle do retificador trifásico proposto. $\mathrm{Na}$ seção V são indicados procedimentos de projeto. Os resultados obtidos experimentalmente estão apresentados na seção VI.

\section{TOPOLOGIA PROPOSTA}

A topologia proposta está representada na Figura 1. Um aspecto interessante desta topologia é poder ser implementada em retificadores já existentes, uma vez que os elementos necessários (interruptores e capacitores) podem ser adicionados ao circuito original, sem necessidade de mudanças significativas.

O papel dos interruptores (que devem ser bidirecionais em tensão e corrente) é fazer com que ocorra uma ressonância entre os indutores de entrada e os capacitores de saída, possibilitando a existência de corrente nas fases durante todo o semiciclo, acompanhando a forma de onda da tensão, conforme mostra a Figura 2.

A topologia de retificador trifásico proposta apresenta um reduzido valor para as capacitâncias $\mathrm{C}_{1}$ e $\mathrm{C}_{2}, \quad\left(\mathrm{C}_{1}=\mathrm{C}_{2}\right)$ responsáveis pela ressonância com o indutor de entrada $\left(\mathrm{L}_{1}=\mathrm{L}_{2}=\mathrm{L}_{3}\right)$ durante os intervalos de chaveamento. $\mathrm{O}$ capacitor de saída, $\mathrm{C}_{\mathrm{O}}$, em relação ao conversor Curi, apresenta, para um mesmo ripple de saída, metade do valor da capacitância.

Como parâmetro de qualidade, foram considerados os limites fornecidos pela IEC 61000-3-4 apresentados na Tabela I: "Estágio 1: Valores de emissão de corrente para conexão simplificada de equipamentos $\left(\mathrm{S}_{\text {equ }} \leq \mathrm{S}_{\mathrm{sc}} / 33\right)$ )", que é válida quando a potência aparente da carga é menor que $3 \%$ da potência de curto circuito do alimentador. Cada limite harmônico de corrente é especificado como função da componente fundamental.

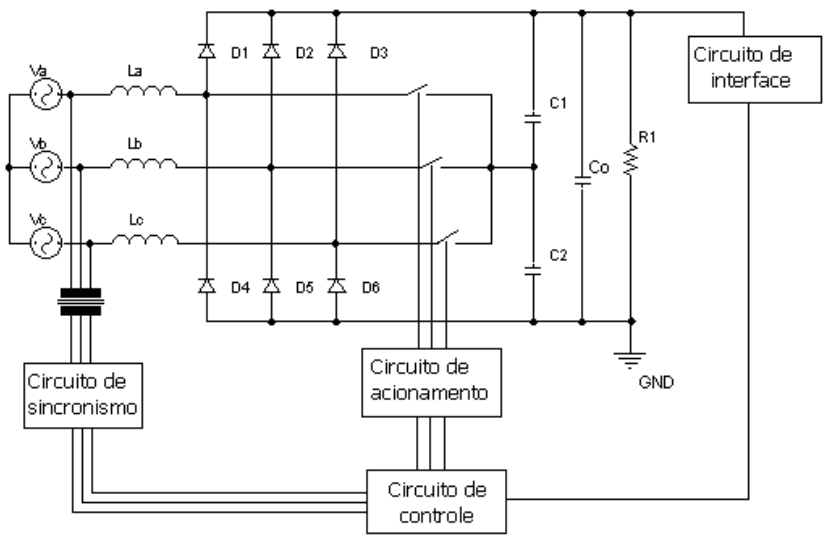

Figura 1 - Topologia proposta com diagrama de controle.

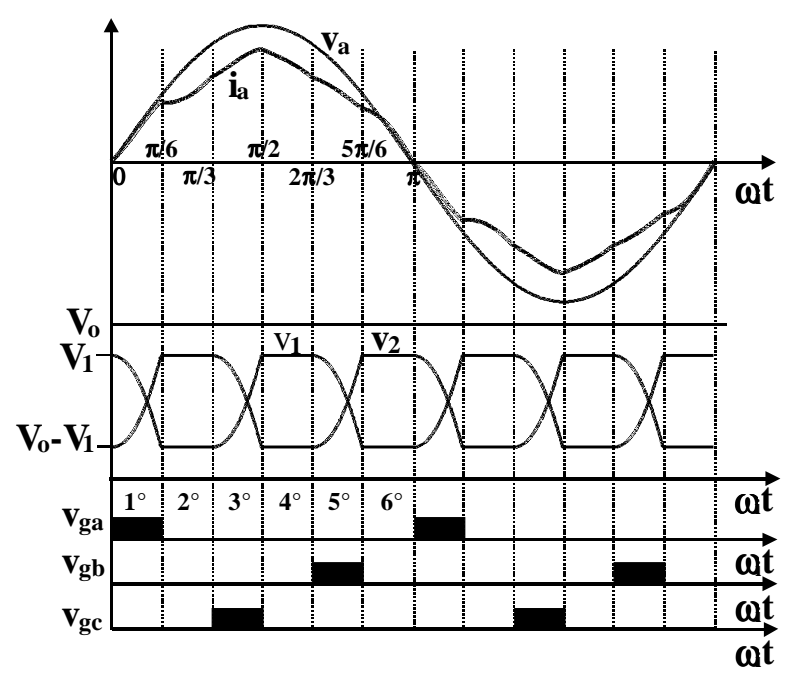

Figura 2 - De cima para baixo: Tensão da fase a; Corrente da fase a; Tensão de saída; Tensões nos capacitores $\mathrm{C}_{1}$ e $\mathrm{C}_{2} \mathrm{e}$ sinais de comando para interruptores. 
Tabela I - "Valores de emissão de corrente para conexão simplificada de equipamentos $\left(\mathrm{S}_{\mathrm{equ}} \leq \mathrm{S}_{\mathrm{sc}} / 33\right) "$

\begin{tabular}{cccc}
\hline \hline $\begin{array}{c}\text { Ordem } \\
\text { harmônica } \\
\mathbf{n}\end{array}$ & $\begin{array}{c}\text { Corrente harmônica } \\
\text { admissível } \\
\mathbf{I}_{\mathbf{n}} / \mathbf{I}_{\mathbf{1}}{ }^{*} \mathbf{\%}\end{array}$ & $\begin{array}{c}\text { Ordem } \\
\text { harmônica } \\
\mathbf{n}\end{array}$ & $\begin{array}{c}\text { Corrente harmônica } \\
\text { admissível } \\
\mathbf{I}_{\mathbf{n}} / \mathbf{I}_{\mathbf{1}}{ }^{*} \mathbf{\%}\end{array}$ \\
\hline 3 & 21,6 & 21 & $\leq 0,6$ \\
5 & 10,7 & 23 & 0,9 \\
7 & 7,2 & 25 & 0,8 \\
9 & 3,8 & 27 & $\leq 0,6$ \\
11 & 3,1 & 29 & 0,7 \\
13 & 2 & 31 & 0,7 \\
15 & 0,7 & $\geq 33$ & $\leq 0,6$ \\
17 & 1,2 & & $\leq 8 / \mathrm{n}$ ou $\leq 0,6$ \\
19 & 1,1 & Ordem par & \\
\hline \hline
\end{tabular}

Os interruptores utilizados manobram uma pequena fração da potência total do conversor. Sua operação em baixa freqüência praticamente elimina as perdas de chaveamento.

Em [12] pode-se encontrar a análise matemática e o método utilizado para projeto dos componentes deste retificador.

A comutação confere à topologia a capacidade de elevar a tensão de saída acima dos valores que seriam obtidos simplesmente com o filtro passivo.

O valor da elevação da tensão depende da indutância de entrada e do intervalo de condução dos interruptores. Controlando-se este intervalo é possível regular a tensão de saída.

\section{IMPLEMENTAÇÃO}

\section{A. Circuito de Sincronismo}

Sua função é detectar a passagem da tensão de cada uma das fases da rede elétrica por zero e sinalizar ao microcontrolador para que este possa gerar os pulsos de comando adequados.

As tensões da rede elétrica são obtidas através de um transformador trifásico que isola e reduz o valor da tensão de entrada. Existe um circuito de sincronismo para cada fase.

A detecção dos momentos da passagem das tensões de fase por zero é feita através de comparadores analógicos. Os ruídos de alta freqüência são descartados por um filtro RC sem inserir atraso significativo.

\section{B. Circuito de Interface da Tensão de Saída}

Este circuito faz a adaptação do nível da tensão de saída do conversor, a qual será amostrada, a um nível de tensão de entrada admissível ao conversor analógico-digital do microcontrolador, que é de $5 \mathrm{~V}$.

Como o sinal de saída apresenta um ripple em $360 \mathrm{~Hz}$, foi necessário implementar um filtro passa-baixas de primeira ordem para atenuá-lo, com freqüência de corte do filtro de $36 \mathrm{~Hz}$.

\section{Circuito de Controle}

Este circuito é responsável pelos cálculos e pela geração dos pulsos de comando dos interruptores e foi implementado com o microcontrolador PIC 17c756a.

O sinal da tensão de saída é subtraído da referência e o erro resultante é aplicado a um controlador do tipo proporcional-integral $(\mathrm{PI})$. O sinal de controle resultante irá determinar o momento do início de condução dos interruptores e o tempo que estes permanecerão conduzindo, entre 0 e $\pi / 6$, para obter a tensão de saída desejada. A informação de cruzamento por zero é proveniente do circuito de sincronismo.

\section{Circuito de Acionamento}

Sua função é acionar os interruptores, seguindo o comando do circuito de controle. Para fazer a isolação dos interruptores em relação ao microcontrolador foram utilizados isoladores ópticos.

Os interruptores auxiliares $\mathrm{Sa}, \mathrm{Sb}$ e Sc são bidirecionais em tensão e corrente, formados por uma ponte retificadora monofásica SKB 15/04 e IGBT IRG4PC50UD. Permite que a corrente flua em ambos os semiciclos, possibilitando a troca de energia entre os indutores de entrada e os capacitores. A freqüência de comutação dos IGBTs é de $120 \mathrm{~Hz}$, com tempo de condução máximo de $1,388 \mathrm{~ms}$, que corresponde a $30^{\circ}$ do ciclo da rede.

\section{ASPECTOS GERAIS DO CONTROLE}

O controle deste retificador foi feito com o uso do microcontrolador PIC 17c756a. Embora nem todos os recursos disponíveis sejam necessários para esta aplicação, sua utilização levou em conta a uniformização de plataformas usadas em vários projetos em andamento no laboratório. Para melhor compreensão o diagrama em blocos do controle é apresentado na figura $3 \mathrm{e}$, a seguir, tem-se uma breve explicação de cada uma das etapas.

\section{A. Inicialização das variáveis}

A primeira etapa do controle digital é a inicialização de algumas variáveis, calculadas previamente, que permitirão o correto funcionamento do retificador, a habilitação ou desabilitação das interrupções, a configuração do timer e das portas de entrada/saída (I/O).

\section{B. Leitura da tensão de saída}

Após a inicialização do programa e sempre que um novo ciclo de programa se inicia, o conversor analógico/digital faz a leitura da tensão de saída.
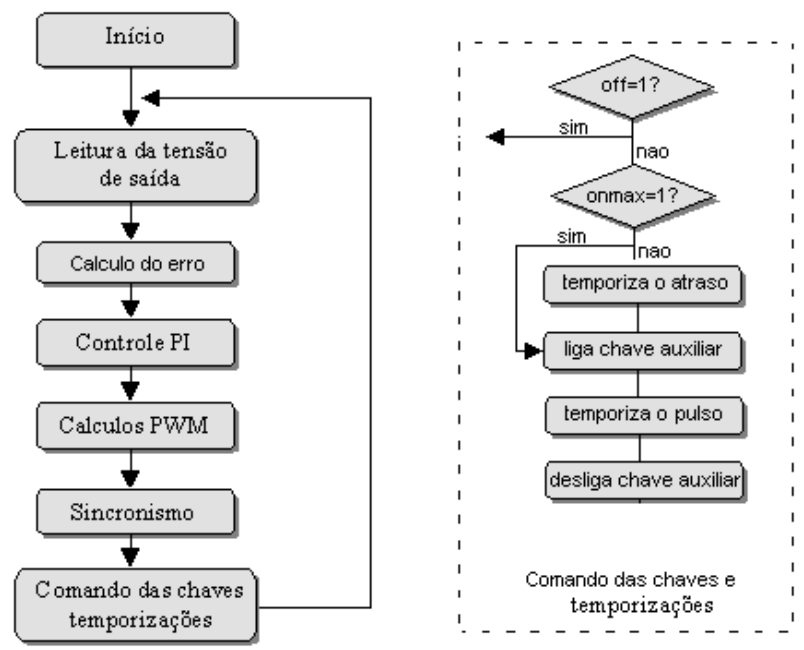

Figura 3 - Diagrama de Blocos do controle. 


\section{Compensador PI}

O compensador escolhido para ser implementado é do tipo proporcional-integral.

A tensão de erro Ve é calculada a partir da tensão de saída $V s$, obtida pelo conversor A/D e a tensão de referência Vref, previamente determinada e inserida na rotina de inicialização.

É gerada uma variável de controle $V c$ que será usada para os cálculos da largura de pulso do comando.

\section{Cálculo da largura de pulso}

Para o controle dos interruptores optou-se pela modulação por largura de pulso (PWM) para variarmos o tempo em que os interruptores permanecem conduzindo.

O sinal de controle dos interruptores é obtido de forma análoga à comparação do sinal de controle proveniente do controlador PI com uma onda portadora triangular, de freqüência $\mathrm{f}=360 \mathrm{~Hz}$. Considerando que as tensões faseneutro nas três fases evoluem na seqüência $\mathrm{Va}, \mathrm{Vb}$ e $\mathrm{Vc}$, os interruptores serão disparados na seqüência $\mathrm{Sa}, \mathrm{Sc}$ e $\mathrm{Sb}$, conforme mostra a figura 2 .

A estratégia de controle adotada para regular a tensão de saída atrasa o instante de condução dos interruptores em relação ao instante do cruzamento da respectiva tensão de fase por zero, enquanto que o instante em que deixam de conduzir é mantido constante.

Após os cálculos necessários para a modulação, são feitas duas verificações:

1. se existe a necessidade de condução de um dos interruptores auxiliares, pois para cargas leves a tensão de saída tende a aumentar, sendo necessário diminuir ou até mesmo suprimir o tempo de condução dos interruptores.

2. se existe atraso, em relação ao cruzamento por zero da tensão, no instante de condução dos interruptores, pois na potência nominal o tempo de condução é máximo e os interruptores auxiliares passam a conduzir a partir do instante do cruzamento por zero.

\section{E. Comando dos interruptores e temporizações}

Optou-se por fazer o sincronismo através de um circuito externo, que envia essa informação ao microprocessador.

Após o programa receber o sinal de sincronismo, verificase se deve ou não ser ligado um interruptor auxiliar. Caso não seja necessário, o programa volta para o início de um novo ciclo. Em seguida verifica-se também se um dos interruptores deve conduzir pelo tempo máximo. Caso seja necessário, o programa pula para o comando de ligar o interruptor, senão o temporizador é carregado com o valor correspondente ao atraso de comutação do interruptor auxiliar e é acionado.

\section{PROCEDIMENTO DE PROJETO}

A definição do valor das indutâncias de entrada e dos capacitores ressonantes, conforme descrito em detalhes [12], utiliza o seguinte procedimento:

Sejam $\alpha=\frac{\omega_{0}}{\omega}, \omega_{0}=\frac{1}{\sqrt{3 L C}}$ e $\omega$ a freqüência angular da rede.
1. Escolha do valor de $\alpha$, entre os valores de $\alpha_{\min }$ e $\alpha_{\max }$. O valor mínimo é aquele que garante que todos os harmônicos obedecem ao limite da IEC 61000-3-4, para qualquer potência, enquanto o valor máximo é dado pela excursão completa da tensão nos capacitores ressonantes. $\alpha_{\max }=3,952$ e $\alpha_{\min }=1,95$ [12].

2. Cálculo da indutância de entrada necessária para a potência de entrada $\mathrm{P}_{\text {in }}$

$$
\begin{gathered}
P_{i n}=\frac{3}{\pi} \int_{0}^{\pi} v_{a}(\theta) \cdot i_{a}(\theta) d \theta=\frac{3 V_{p}^{2}}{\pi \omega L} \int_{0}^{\pi} v_{a n}(\theta) \cdot i_{a n}(\theta) d \theta=\frac{V_{p}^{2}}{\omega L} P_{i n N} \\
L=\frac{V_{p}^{2}}{\omega P_{i n}} P_{i n N}
\end{gathered}
$$

onde:

$v_{a n}$ é a tensão de entrada normalizada em relação ao seu valor de pico $\left(V_{p}\right)$.

$i_{a n}$ é a corrente de entrada normalizada em relação a $\left(V_{p} / \omega L\right)$.

$P_{i n N}$ é a potência de entrada normalizada em relação a $\left(V_{p}^{2} / \omega L\right)$, sendo função apenas do parâmetro $\alpha$, e pode ser obtida da curva mostrada na figura 4.

3. Cálculo dos capacitores ressonantes a partir dos valores de $\alpha$ e L

4. Verificar a tensão média de saída máxima $\left(\mathrm{V}_{\mathrm{Omax}}\right)$;

$V_{O \max }=\frac{18 V_{p}}{7 \pi}\left\{1+\frac{1}{\left(\alpha^{2}-1\right)}\left[\frac{\alpha^{2} \sqrt{3}}{2}-\cos \left(\frac{\alpha \pi}{6}\right)+F(\alpha) \operatorname{sen}\left(\frac{\alpha \pi}{6}\right)\right]\right\}$ (3) onde $F(\alpha)=\frac{\frac{\alpha}{2}-\sin \left(\alpha \frac{\pi}{6}\right)}{1+\cos \left(\alpha \frac{\pi}{6}\right)}$

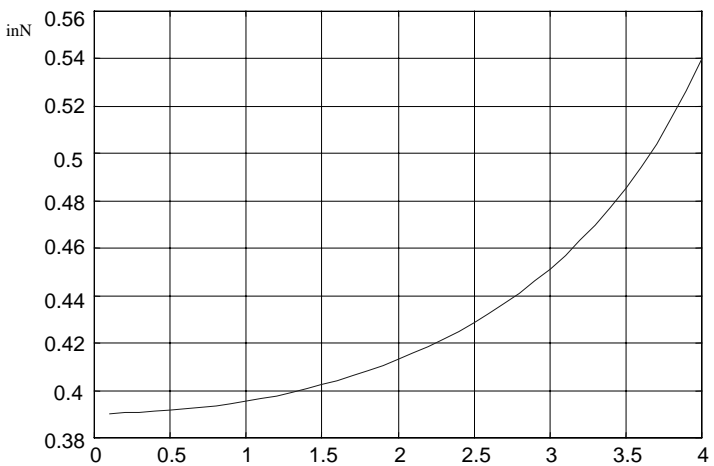

Figura 4 - Potência de entrada normalizada em função de $\alpha$.

\section{RESULTADOS EXPERIMENTAIS}

O retificador foi projetado para uma tensão de fase de $127 \mathrm{~V}$, potência de saída de $9,6 \mathrm{~kW}$ e rendimento estimado de $97 \%$, resultando numa potência de entrada de $9,9 \mathrm{~kW}$.

O valor da indutância para a potência desejada é $\mathrm{L}=4,2 \mathrm{mH}$, e os capacitores de ressonância serão $\mathrm{C}=42,7 \mu \mathrm{F}$, para $\alpha=3,6$. Estes são compostos de capacitores ligados em paralelo. $\mathrm{O}$ uso de vários capacitores em paralelo permite reduzir a resistência série equivalente, minimizando as perdas nestes componentes. 
O capacitor de saída é de $800 \mu \mathrm{F}$ / 400V. A capacitância de saída pode ser calculada em função da ondulação de tensão admissível, como é normalmente feito no projeto de retificadores. No presente circuito, quando se tem o funcionamento do circuito auxiliar, a ondulação será reduzida em relação a este valor.

Com este valor de potência, a corrente eficaz de entrada esperada é de $25 \mathrm{~A}$. A ponte retificadora trifásica utilizada é SKD 50/12 com capacidade de corrente de 50A.

Pelos interruptores auxiliares, $\mathrm{Sa}, \mathrm{Sb}$ e $\mathrm{Sc}$, calcula-se, na máxima condução, que passe uma corrente eficaz de $3,4 \mathrm{~A}$, com pico de 22,2A. São compostos de ponte retificadora monofásica SKB 15/04 e IGBT IRG4PC50UD, com capacidade de corrente de 27A, disponíveis no laboratório.

Foram feitos ensaios com diferentes níveis de potência para verificação do conteúdo harmônico da corrente de entrada, conforme determina a IEC 61000-3-4. Os resultados estão apresentados na Tabela II.

Na figura 5, tem-se a tensão e a corrente de entrada do retificador na potência nominal e na figura 6 para aproximadamente $40 \%$ da potência nominal.

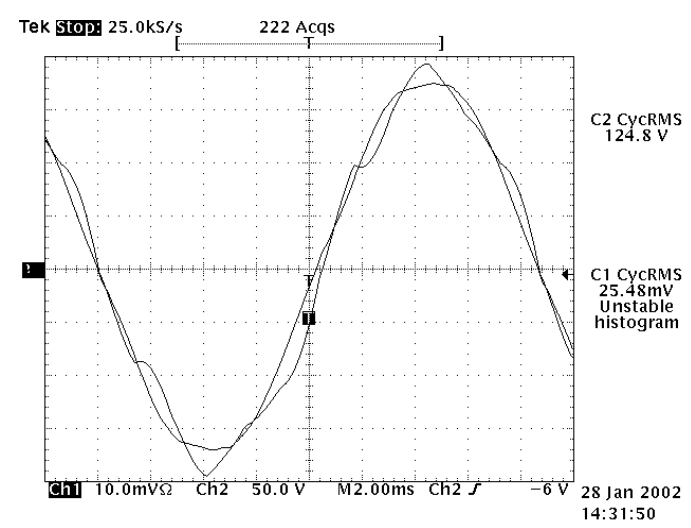

Figura 5 - Tensão de entrada (50V/div.) e corrente (10A/div.) para $\mathrm{P}_{\text {in }}=9828 \mathrm{~W}$.

Tabela II - Conteúdo harmônico da corrente de entrada normalizado em relação à componente fundamental

\begin{tabular}{c|cccccc}
\hline \multicolumn{7}{c}{ Corrente (A) para diferentes níveis de } \\
potência & Limites \\
$\mathbf{P}_{\text {in }}(\mathbf{W})$ & $\mathbf{9 8 2 8}$ & $\mathbf{8 4 8 4}$ & $\mathbf{5 8 2 0}$ & $\mathbf{3 7 2 0}$ & $\mathbf{9 3 0}$ & IEC1000-3-4 \\
\hline $\mathbf{1}$ & 25,3 & 22,3 & 16,64 & 10,2 & 2,46 & - \\
$\mathbf{3}$ & 0,15 & 0,136 & 0,12 & 0,032 & 0,082 & 5,4648 \\
$\mathbf{5}$ & 2,03 & 1,51 & 0,444 & 0,728 & 0,578 & 2,7071 \\
$\mathbf{7}$ & 0,432 & 0,476 & 1,28 & 1,252 & 0,556 & 1,8216 \\
$\mathbf{9}$ & 0,01 & 0,014 & 0 & 0,032 & 0,018 & 0,9614 \\
$\mathbf{1 1}$ & 0,33 & 0,462 & 0,572 & 0,528 & 0,15 & 0,7843 \\
$\mathbf{1 3}$ & 0,276 & 0,278 & 0,204 & 0,204 & 0,196 & 0,506 \\
$\mathbf{1 7}$ & 0,084 & 0,062 & 0,156 & 0,316 & 0,084 & 0,3036 \\
$\mathbf{1 9}$ & 0 & 0,138 & 0,128 & 0,23 & 0,116 & 0,2783 \\
$\mathbf{2 3}$ & 0,062 & 0,102 & 0,016 & 0,032 & 0,046 & 0,2277 \\
$\mathbf{2 5}$ & 0,062 & 0,068 & 0,028 & 0,072 & 0,058 & 0,2024 \\
$\mathbf{2 9}$ & 0,022 & 0,046 & 0 & 0,026 & 0,032 & 0,1771 \\
$\mathbf{3 1}$ & 0 & 0,072 & 0 & 0,018 & 0,04 & 0,1771 \\
$\mathbf{3 5}$ & 0,014 & 0,036 & 0,04 & 0,046 & 0,032 & 0,1518 \\
$\mathbf{3 7}$ & 0,026 & 0,042 & 0,04 & 0,022 & 0,032 & 0,1518 \\
\hline \hline
\end{tabular}

O gráfico de regulação de tensão para um ajuste de 297V está na figura 7.

À medida que se diminui a carga para valores abaixo de $1 \mathrm{~kW}$ a tensão de saída tende a subir, pois o tempo que os interruptores permanecem conduzindo se anula e o circuito perde sua capacidade de regulação.

Para uma carga nula, conversor a vazio, esta tensão tende ao valor de pico da tensão de linha, ou seja, $311 \mathrm{~V}$.

Quando se atinge a máxima largura de pulso do comando em potência elevada, o sistema não mais consegue manter a regulação. De acordo com a figura 7 , na faixa de $10 \%$ a $100 \%$ da potência nominal a regulação é de $0,8 \%$.

Na Tabela III pode-se observar a taxa de distorção harmônica da corrente de entrada, o fator de potência e o fator de deslocamento $\left(\cos \varphi_{1}\right)$, para diferentes níveis de potência.

Pode-se observar que o valor do fator de deslocamento é sempre próximo de um, pois a estratégia de controle adotada faz com que a componente fundamental da corrente de entrada esteja praticamente em fase com a tensão.

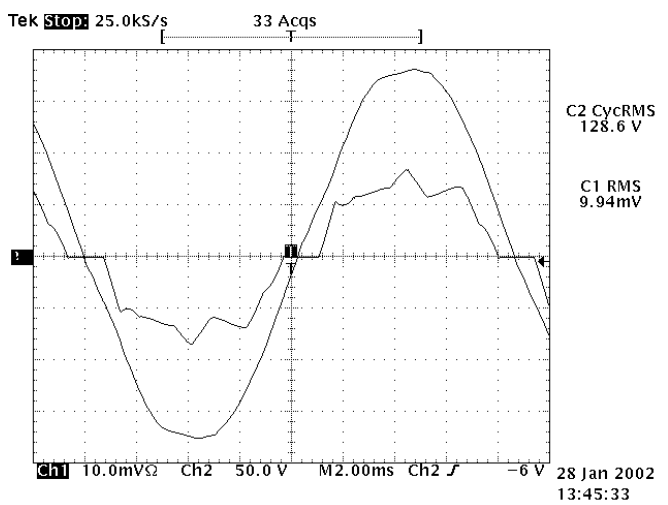

Figura 6 - Tensão de entrada (50V/div.) e corrente (10A/div.) para $\mathrm{P}_{\text {in }}=3720 \mathrm{~W}$.

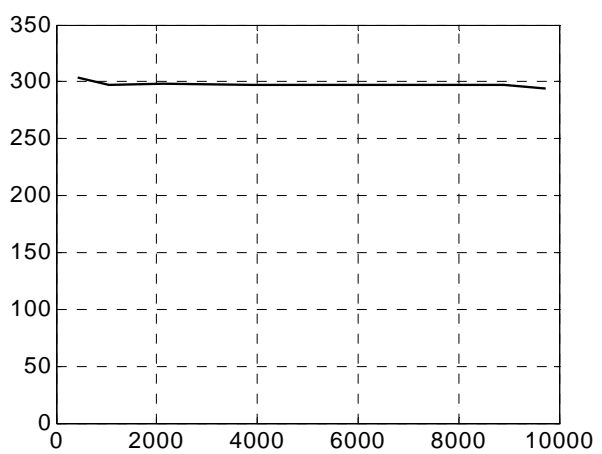

Figura 7 - Regulação da tensão de saída (valor nominal de 297V) em função da potência de saída.

Tabela III - Distorção da corrente de entrada, fator de deslocamento e fator de potência para diferentes níveis de potência

\begin{tabular}{c|cccc}
\hline \hline $\mathbf{P}_{\mathbf{i}}(\mathbf{W})$ & $\mathbf{I}_{\mathbf{i}}\left(\mathbf{A}_{\mathbf{R M S}}\right)$ & $\mathbf{T H D}(\boldsymbol{\%})$ & $\cos \boldsymbol{\varphi}_{\mathbf{1}}$ & $\mathbf{F P}$ \\
\hline $\mathbf{9 3 0}$ & 2,67 & 46,2 & 0,99 & 0,88 \\
$\mathbf{1 8 3 0}$ & 4,97 & 30,6 & 0,99 & 0,94 \\
$\mathbf{3 7 2 0}$ & 9,77 & 17,1 & 0,99 & 0,98 \\
$\mathbf{5 8 2 0}$ & 15,0 & 10,8 & 1,00 & 0,99 \\
$\mathbf{8 4 0 0}$ & 22,0 & 8,3 & 1,00 & 0,99 \\
$\mathbf{9 6 0 0}$ & 25,2 & 8,6 & 1,00 & 0,99 \\
\hline \hline
\end{tabular}




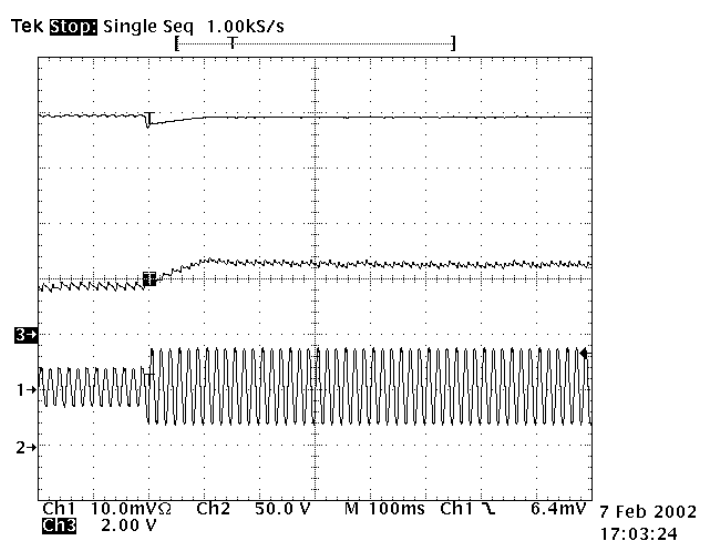

Figura 8 - Tensão de saída (50V/div), tensão de controle do PI ( $2 \mathrm{~V} / \mathrm{div})$ e corrente de entrada (20A/div), tempo (100ms/div)

Para valores baixos de corrente de carga, o retificador apresenta maior taxa de distorção harmônica da corrente.

$\mathrm{O}$ fator de potência observado apresenta bons valores para potências elevadas, próximos a 0,99. Para uma carga de aproximadamente $10 \%$ da potência nominal, seu valor é 0,88 .

Foram feitas variações na carga para observar o comportamento do controle. O PI implementado apresenta os seguintes valores: $\mathrm{Kp}=0,375$ e $\mathrm{Ti}=1,388 \mathrm{~ms}$, que foram ajustados empiricamente, pois não foi objetivo deste trabalho o desenvolvimento de um modelamento dinâmico do conversor, o que permitiria estabelecer um método de projeto do sistema de controle.

Na figura 8 tem-se uma variação de carga de 930W para 1830W. Pode-se observar uma rápida variação na corrente de entrada. A tensão de saída sofre um pequeno decréscimo e retorna para seu valor nominal, próximo de $300 \mathrm{~V}$. O sinal de controle do regulador $P I$ atinge seu novo valor de regime em $150 \mathrm{~ms}$.

À medida que se varia a potência de saída, o controle varia o atraso no instante de entrada em condução dos interruptores auxiliares e o tempo que estes permanecem conduzindo. Conseqüentemente, os valores máximos e mínimos de tensão observados nos capacitores de ressonância também variam.

Na figura 9 tem-se as formas de onda da tensão de saída e em um dos capacitores de ressonância para carga nominal. Neste caso tem-se a máxima excursão da tensão sobre o capacitor nos instantes de ressonância. A tensão inicial no capacitor $\mathrm{V}_{\mathrm{C} 1}$, em regime permanente, é de $60 \mathrm{~V}$, elevando-se a $220 \mathrm{~V}$.

\section{VII.CONCLUSÕES}

A topologia proposta apresenta boas características para compensar distorções na corrente produzidas por pontes retificadoras a diodos com filtro capacitivo e apresenta vantagens em relação às demais alternativas apontadas na bibliografia.

O uso de baixa freqüência de comutação minimiza as perdas, melhorando o rendimento. Os componentes harmônicos de correntes resultantes estão abaixo dos limites especificados no relatório técnico IEC 61000-3-4. Os valores dos elementos magnéticos são reduzidos, quando comparados com soluções puramente passivas, para o atendimento dos mesmos limites.

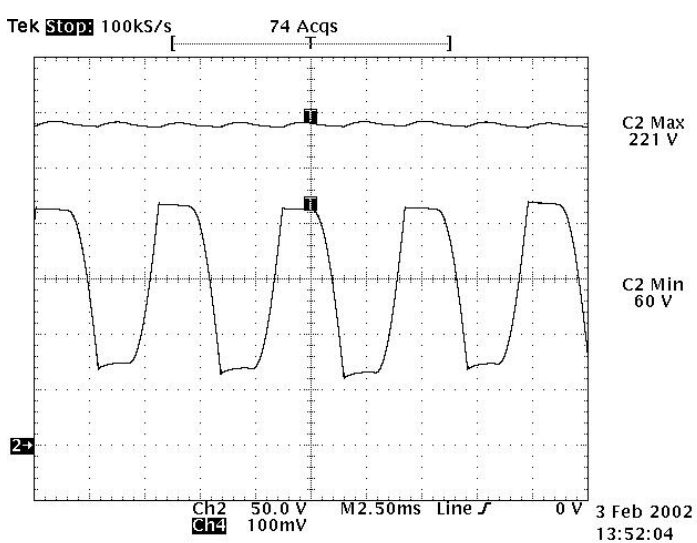

Figura 9 - Tensão de saída e no capacitor $C_{1}(50 \mathrm{~V} /$ div.) para potência nominal.

Pelos resultados gerais obtidos pode-se concluir que a topologia proposta permite adequar os retificadores trifásicos às limitações de distorções harmônicas da corrente estabelecidas por organismos internacionais.

A possibilidade de inclusão de o circuito auxiliar em retificadores já existentes não exige a substituição do conversor CA-CC, como seria necessário com o uso de conversores PWM.

Além da melhoria na forma de onda da corrente, o circuito auxiliar permite uma elevação na tensão de saída e sua regulação.

\section{AGRADECIMENTOS}

Este projeto foi financiado pela FAPESP, a quem os autores agradecem.

\section{REFERÊNCIAS BIBLIOGRÁFICAS}

[1] T. Tanaka, N. Koshio, H. Akagi, A. Nabae: "Reducing Supply Current Harmonics". IEEE Industry Applications Magazine, pp. 31-37, Sep./Oct. 1998.

[2] International Electrotechnical Commission: 61000-3-2 "Limits for Harmonic Currents Emission (Equipment input current up to and including 16A per phase)", first edition, 1995.

[3] International Electrotechnical Commission: 61000-3-4 "Limitation of emission of harmonic currents in lowvoltage power supply systems for equipment with rated current greater than 16A", first edition, 1998.

[4] J. A. Pomílio, G. Spiazzi, S. Buso. "Comparison among High-Frequency and Line-Frequency Commutated Rectifiers Complying with IEC 61000-3-2 Standards", IEEE Industry Applications Society Annual Meeting (IAS'2000), Proceedings pp. 2218-2223.

[5] I. Rastogi, R. Naik, N. Mohan, "Optimization of a Novel DC-Link Current Modulated Interface with 3-Phase Utility Systems to Minimize Line Current Harmonics," IEEE Power Electronics Specialists Conference (PESC'92), Proceedings pp.162-167.

[6] N. Mohan, "A Novel Approach to Minimize LineCurrent Harmonics in Interfacing Renewable Energy Sources with 3-Phase Utility Systems," IEEE Applied Power Electronics Conference (APEC'92), Proceedings pp. 852-858, Boston, 1992. 
[7] S. Hansen, P. N. Enjeti, J-H. Hahn, F. Blaabjerg, “An Integrated Single-Switch Approach to Improve Harmonic Performance of Standard PWM AdjustableSpeed Drives", IEEE Transactions on Industry Applications, Vol. 36, NO.4, July/August, 2000, pp. 1189-1196.

[8] G. Kamath, B. Runyan and R. Wood "A Compact Autotransformer based 12-Pulse Rectifier Circuit" ", IEEE Industrial Electronics Conference (IECON'01), CD-ROM.

[9] I. Barbi, J. C. Fagundes and C. M T. Cruz: "A Low Cost High Power Factor Three-Phase Diode Rectifier with Capacitive Load". IEEE Applied Power Electronics Conference (APEC'94) Proceedings pp. 731-737, March 1994.

[10]E. L. M. Mehl and I. Barbi, “An Improved High Power Factor and Low Cost Three-Phase Rectifier", IEEE Applied Power Electronics Conference (APEC'95) Proceedings, pp. 1-7, March 1995.

[11]J. W. Kolar and F. C. Zach " A novel three-phase, three switch, three level unity power factor PWM rectifier", $28^{\text {th }}$. PCIM Conf., Nürnberg, Germany, 1994.

[12]J. A. G. Marafão, J. A. Pomilio, G. Spiazzi “A HighQuality Three-Phase Rectifier complying with IEC 61000-3-4 Standards", $6^{\text {th }}$ Brazilian Power Electronics Conference (COBEP'2001), CD-ROM.

\section{DADOS BIOGRÁFICOS}

Joanna Aboin Gomes Marafão, nascida em 22/04/1976 em Campinas (SP) é engenheira eletricista (1999) pela Universidade Estadual Paulista e mestre em Engenharia Elétrica (2002) pela Universidade Estadual de Campinas. Em 2002 realizou um estágio junto à Universidade de Pádua, Itália.

José Antenor Pomilio, nascido em 06/05/1960 em Jundiaí (SP), é engenheiro eletricista (1983), mestre (1986) e doutor em Eng. Elétrica (1991) pela Universidade Estadual de Campinas, onde é docente desde 1984. De 1988 a 1991 foi chefe do grupo de eletrônica de potência do Lab. Nacional de Luz Síncrotron. Em 1993/1994 realizou estágio de pósdoutoramento junto à Universidade de Pádua - Itália. Foi presidente da SOBRAEP (2001/2002). Suas áreas de interesse são fontes de alimentação, qualidade de energia e acionamento de máquinas elétricas.

Giorgio Spiazzi nasceu em Legnago (província de Verona, Itália) em 1962. Graduou-se em Engenharia Elétrica na Universidade de Padova em 1988. Em 1993 obteve seu doutorado em Eletrônica Industrial e Informática no Departamento de Eletrônica e Informática da mesma Universidade, onde é professor desde 1993. Suas áreas de interesse são técnicas de controle avançadas para conversores de potência, pré-conversores de alto fator de potência e técnicas de comutação suave. 